\title{
Multiplicative method for creating the traffic monitoring base in a megapolis
}

\author{
Olga Makovetskaya-Abramova ${ }^{1}$, Yuriy Lazarev ${ }^{1}$, Marina Gravit, Svetlana Silla ${ }^{1}$, and \\ Mariya Shakhova ${ }^{1, *}$ \\ ${ }^{1}$ Peter the Great St. Petersburg Polytechnic University, 29, Polytechnicheskaya, 195251, Saint \\ Petersburg, Russia
}

\begin{abstract}
The purpose of the study is the development of methods of identification of vehicles. These methods based on the system of identification their optical images for organization of movement and reducing the number of road accidents. There is a way of mathematical processing of signals in this method. These signals are registered with the help of optical-electronic sensors for the rapid decision-making based on computer technology. As a result, there is a confirmation of the method's operability in real conditions. Digital signal processing lets to determine the intensity, speed and density of traffic flow automatically. At the same time, multiplicative method is a method of multidimensional analysis in a multidimensional space of numerical criteria. It divides the traffic flow by classes. This technology opens a lot of great opportunities for optimizing traffic management and helps to improve the safety of vehicles. The results of the survey are proposed for using in smart city systems.
\end{abstract}

\section{Introduction}

The ideas of creating intelligent transport are connected with the concept of creating a smart city [1 - 6]. There is an analysis of the road situation and monitoring of traffic should be carried out in the metropolis. The task of automatic monitoring is reduced to solving detection problems.

Detection is one of the forms of classification. Objects that belong to the same image class have common properties. Classes can be set in advance if the car 's affiliation to the certain class is known beforehand. The simplest approach of detection of images is a comparison with the standard. There is a detailed image that does not coincide with any of the classified standard images in real-world conditions. Nevertheless, there may be a correlation between them. Therefore, criteria derived from correlation theory can be used, and the relationship between the features of the images can be taken as essential features. Correlation is a feature indicating the relationship of the several numerical sequences. The paired correlation characterizes the relationship between the two sequences $\mathrm{x} i$ and yi. The pairwise correlation coefficient $\mathrm{R}$ characterizes the degree of deviation of the relation between $x i$ and yi from the linear relation:

\footnotetext{
*Corresponding author: shahovashahova@yandex.ru
} 


$$
R=\frac{\sum_{i=1}^{N} x_{i} \cdot y_{i}-\left(\sum_{i=1}^{N} x_{i} \cdot \sum_{i=1}^{N} y_{i}\right) / N}{\sqrt{\sum_{i=1}^{N} x_{i}^{2}-\frac{\left(\sum_{i=1}^{N} x_{i}\right)^{2}}{N}} \cdot \sqrt{\sum_{i=1}^{N} y_{i}^{2}-\frac{\left(\sum_{i=1}^{N} y_{i}\right)^{2}}{N}}}
$$

If the module $\mathrm{R}$ is close to 1 , then this relationship is linear, i.e. $\mathrm{y} i=\mathrm{axi}+\mathrm{b}$. So, the sign before $\mathrm{R}$ defines the sign before the coefficient a. Calculation of the cross-correlation of the averaged signals, obtained when the truck raster passes in the field of view with the paired correlation coefficient $\mathrm{R}=0.936$, indicates a close relationship to the optical images of one class of vehicles.

It is very important to select a rational set of characteristics. It allows to identify the presented image with the standard based on any criterion. The presence of a representative set of characteristics allows to apply the multiplicative method.

\section{Methods}

The basis of the multiplication method [7] is a synthesis of criteria (homogeneous complexes of parameters) characterizing a certain system or development of events. The method consists of several steps:

- Finding the main influencing values that play the role of arguments.

- Extraction from noises of these values and their measurement.

- Synthesis of the determinative equation in the form of (2), thereby forming a criterion of interest.

There is a condition of fairness of the principle of absolute relations for laws of physics in any systems of primary quantities is homogeneity of determining equations. But only exponentiation equations are homogeneous:

$$
Y=A x_{1}^{\alpha 1} \cdot x_{2}^{\alpha 2} \cdot \ldots \cdot x_{k}^{\alpha k}
$$

Therefore, the search for physical relations between different values must be carried out by constructing the equation as (2). The values of the quantities are called dimension measures or the dimension of the secondary quantity. This approach to research of physical processes is called Dimensional Analysis [7].

In case of using the multiplication method of statistical processing, the observation table has only a particular role. The calculation of the pair correlation coefficients is facilitating the search for arguments, i.e. the main influencing factors. The creation of a quantity unit is reduced to the following operations:

1. The correlation analysis is closely relation these values (taken in different degrees) and the parameter with determined unit of measure.

2. The defining product is generated, and the unit of measure is set.

3. Reliability of the unit's measure is checked by calculating the correlation coefficient between the generated product and the analyzed parameter. This coefficient should be close to 1 , which provides an almost functional relationship between these values. 


\section{Results and discussions}

Suppose there is a multidimensional feature space (criteria space), each of which is part of the equation of type (2):

In the feature space, each vehicle occupies some volume. For a class, this volume has odd boundaries. In case the volumes do not intersect (Fig. 1, a), the division of vehicle into classes by the size of the defining products is not difficult. If the volumes overlap partially (Fig. 1, b), which is equivalent to mathematical operation "AND," then the task of division into classes becomes more complicated. In this case, it is necessary to calculate the probability of correct detection and false alarm. The number of criteria in the defining product, and thus the complexity of the algorithm applied, is chosen from the permissible probabilities and cost of errors. The optimal number of criteria is 7 [7].

a)

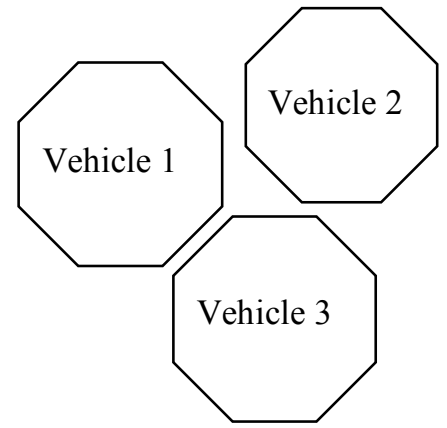

b)

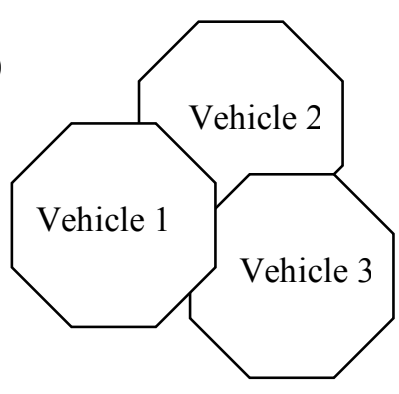

Fig. 1. a) Volumes occupied by individual vehicle classes do not overlap in multidimensional feature space; b) Partial intersection of volumes occupied by individual vehicle classes - complicating the problem of image detection in multidimensional feature space.

Figure 2 below shows a graph of the probability density function of the values of the defining products by vehicle classes.

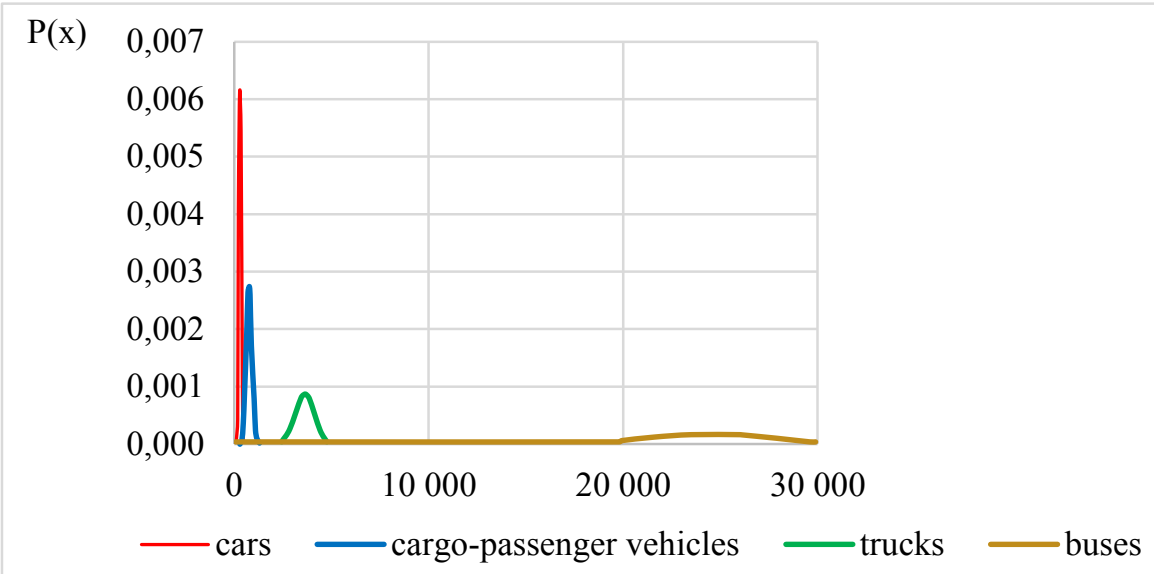

Fig. 2. Theoretical distribution of probability density function of the values of the defining products by vehicle classes.

The value of the defining product differs tenfold for car class and bus class:

$$
y=\Delta t \cdot \bar{A}_{\max }^{2}
$$


where $\Delta t$ - time of vehicle passing in raster field of view;

$\bar{A}_{\text {max }}^{2}$ - maximum signal strength.

Figure 3 shows that the frequency boundaries of the class division are clear, which makes it possible to predict a high degree of reliability of identification of vehicles by class by the multiplication method.

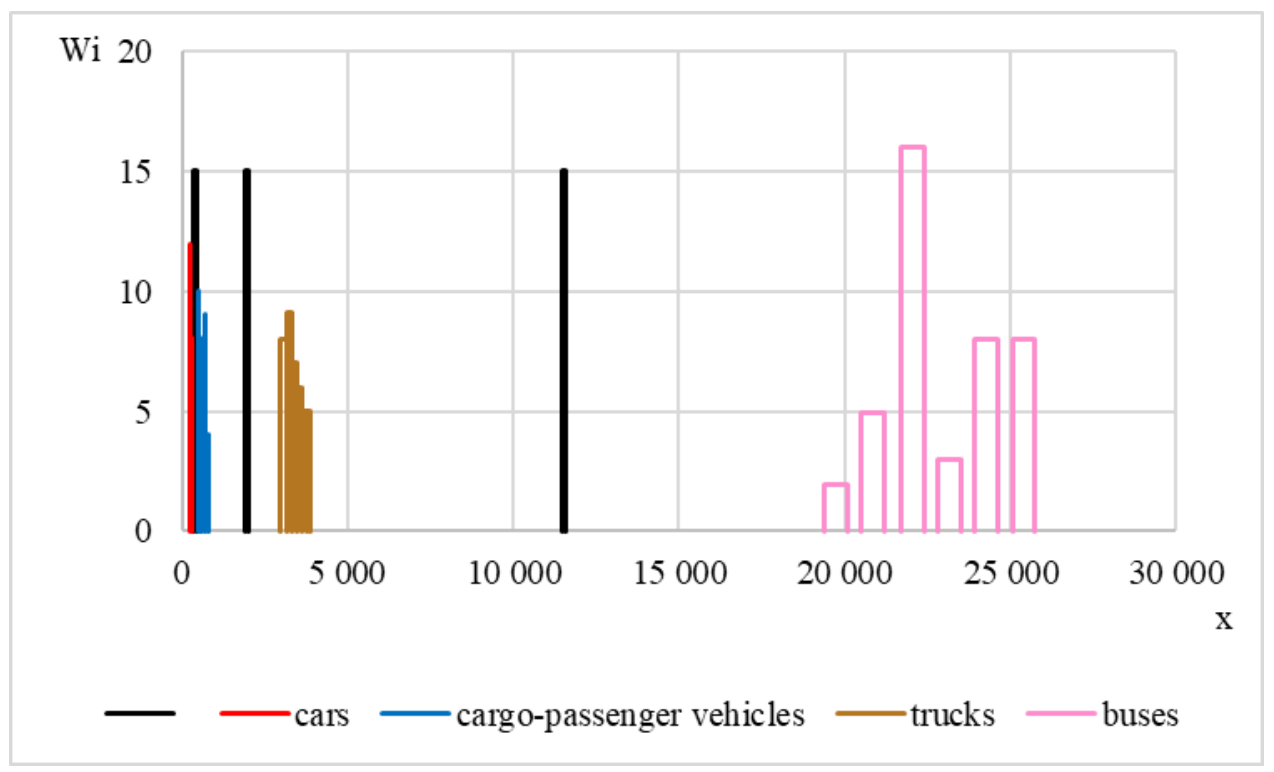

Fig. 3. Histogram of frequencies of objects. Result of search of vehicle class boundaries.

As mutually independent indicators (main influencing factors) the product of amplitude maximum $\mathrm{i}$ (maximum signal intensity) and maximum value along abciss axis $\mathrm{t}$ (signal existence time) is selected, as well as direct and inverse ratio t to $i$.

Analysis of the nature of the recognition task under the conditions shown in Figure 1(b), i.e. with a probabilistic relation between object features and vehicle classes, showed that statistical decision theory, or hypothesis-checking theory, created by Neyman and Pearson, could be used with success to construct recognition algorithms. If an object belongs to class I and it is considered an object of class II, an error of the first kind has been made [9]. According to the terminology of statistical decision theory, the $\mathrm{H}_{2}$ hypothesis is wrongly chosen, while the $\mathrm{H}_{1}$ hypothesis is true. The probability of an error of the first kind, that is, the probability of assigning an object to class II when it belongs to class I:

$$
Q_{1}=\int_{X_{0}}^{\infty} f_{1}(X) d X
$$

On the contrary, if the $\mathrm{H}_{2}$ hypothesis is true, and preference is given to the $\mathrm{H}_{1}$ hypothesis, a second kind of error has been made, the probability of which is:

$$
Q_{2}=\int_{-\infty}^{X_{0}} f_{2}(X) d X
$$

$\mathrm{Q}_{2}$ there is a chance of choosing the $\mathrm{H}_{1}$ hypothesis when the $\mathrm{H}_{2}$ hypothesis is true. 
The search for boundaries between classes of vehicles was carried out using the Quest Software [9]. The probability of incorrect conclusion when recognizing vehicles of two neighboring classes (cars and small buses) was:

- by one independent criterion (signal existence time) - 70\%;

- by two independent criteria (product of signal amplitude maximum and signal existence time) $-18 \%$;

- by three independent criteria (the product of the square of the amplitude maximum and signal existence time, which in the physical sense is close to the energy of the signal) $-0 \%$.

The presence of a representative set of features of the optical image allows to apply the multiplication method [10-17]. The basis of the multiplication method is the synthesis of criteria (homogeneous complexes of parameters) characterizing a certain system or development of events. The application of the multiplication method allows to identify vehicles, as the values of defining products differ significantly for cars of different classes (cars, cargo-passenger vehicles, trucks, buses).

Information on the quality of the traffic is necessary for the operation of the smart city transport platform. On the basis of data on intensity and density at speed mode of movement it is possible to automatically adjust modes of operation of traffic light network. With the use of information on classes of vehicle, the justification of funds for repair and construction of roads, construction of roadside infrastructure, logistics management, realtor expertise and assessment of attractiveness for residence, investment and development of districts of the city is connected. Monitoring the environmental situation, safety and comfort on the road.

\section{References}

1. V. Vlasov, I. Konin, P. Pribyl, V. Bogumil, Transport. Res. Proc., 683-689 (2017)

2. V. Bogumil, V. Vlasov, Transport. Res. Proc., 63-67 (2018)

3. S. Kauf, Transport. Res. Proc. 39, 143-149 (2019)

4. C. M. Leobons, V. Campos, R. A. M. Bandeira, Transport. Res. Proc. 37, 322-329 (2019)

5. M. Gravit, I. Dmitriev, Y. Lazarev, EMMFT 2018. 983, 929-938 (2019)

6. J. Zemitis, M. Terekh, EECE - 2018. (2018)

7. T. Szirtes, Applied Dimensional Analysis and Modeling (Butterwotrth-Heinemann, 2007)

8. A. J. Rojas, J. I. Yuz, IFAC Proc. 41, 5179-5184 (2008)

9. T. Sterling, M. Brodowicz, M. Anderson, High Performance Computing (Morgan Kaufmann, 2018)

10. O. Gamayunova, R. Golov, E3S Web of Conferences. 135, 02025 (2019)

11. K. Buka-Vaivade, J. Sliseris, D. Serdjuks, L. Pakrastins, N.I. Vatin 2018. 84(8). 3-14. DOI:10.18720/MCE.84.1.

12. C. Campian, N. Chira, V. Iuhos, M. Pop, N. Vatin. Procedia Engineering. 2016. 165. Pp. 876-882. DOI:10.1016/j.proeng.2016.11.787.

13. A.V. Chechevichkin, N.I. Vatin, V.V. Samonin, M.A. Grekov. Magazine of Civil Engineering. 2017. 76(8) 201-213. DOI:10.18720/MCE.76.18.

14. V. Okrepilov, M. Gravit, E. Nedviga, O. Dudnik, Jour. of Appl. Eng. Scien. 14, 135139 (2016)

15. S. Luca, R. Di Pace, Int. Jour. of Sustain. Transport. 12, 353-366 (2018)

16. M. Gravit, S. Zimin, Y. Lazarev, I. Dmitriev, E. Golub, Spring. Int. Publish. 1116 (2020)

17. M. A. Javed, S. Zeadally, E. B. Hamida, Vehicul. Comm. 15, 63-72 (2019) 